\title{
Time-Domain Modeling of a Distribution System to Predict Harmonic Interaction Between PV Converters
}

\author{
Grazia Todeschini ${ }^{\circledR}$, Senior Member, IEEE, Senthooran Balasubramaniam, Member, IEEE, \\ and Petar Igic ${ }^{(1)}$, Senior Member, IEEE
}

\begin{abstract}
Due to the interaction converter control, pre-existing distortion and grid impedance, the harmonic levels caused by renewable energy sources are continuously changing, and their assessment requires the use of dedicated computer models. Several time-domain models have been proposed to carry out this analysis, however, they fall short of at least one requirement: either they do not provide accurate results, or they require an excessively long simulation time. This paper presents a novel time-domain model to address the gap described above: the proposed model employs average functions and a novel switching emulator. Therefore, it is referred to as "average model with switching emulator" (AMSE). The proposed model is compared with existing models, and the results indicate that the AMSE meet both requirements stated above, as it accurately represents harmonic distortion while reducing significantly the simulation time. The second part of the paper discusses mitigating solutions to harmonic amplification in systems with a high penetration of VSCs, and shows the effectiveness of using an active filter to reduce harmonic levels in system experiencing resonance conditions.
\end{abstract}

Index Terms-Active filter, average model (AM), harmonic analysis, detailed model (DM), photovoltaic (PV) energy, resonance, voltage source converter (VSC), renewable energy source (RES).

\section{INTRODUCTION}

$\mathbf{E}$ NVIRONMENTAL concerns and technological advancements have accelerated the development of renewable energy sources (RESs) and their integration into the power grid. Although RESs are becoming a significant portion of energy generation in many countries, they have some adverse effects on grid operation, including impacts on power quality [1]-[3].

RESs are connected to the grid by means of power converters, and commutation of the power switches results in harmonic current and voltage components. While in the majority of systems

Manuscript received July 9, 2018; revised November 13, 2018; accepted February 14, 2019. Date of publication February 22, 2019; date of current version June 20, 2019. This work was supported in part by the European Region Development Fund and in part by the Welsh Government under the FLEXIS project. Paper no. TSTE-00668-2018. (Corresponding author: Grazia Todeschini.)

G. Todeschini and S. Balasubramaniam are with the College of Engineering, Swansea University, Swansea SA1 8EN, U.K. (e-mail: grazia.todeschini@swansea.ac.uk; S.balasubramaniam@swansea.ac.uk).

P. Igic is with the Coventry University, Coventry CV1 5FB, U.K. (e-mail: p.igic@swansea.ac.uk).

Color versions of one or more of the figures in this paper are available online at http://ieeexplore.iee.org.

Digital Object Identifier 10.1109/TSTE.2019.2901192 the levels of harmonic distortion caused by RESs are within the limits prescribed by the standards [4], [5], the increasing number of non-linear loads and RESs connected to the grid is expected to cause impacts on grid operation in the near future. A survey was carried out among thirty-two utilities in eighteen countries, indicating that power quality is becoming one of the main concerns for future power grids [6].

Predicting the levels of harmonics caused by RESs is complex due to the availability of numerous inverter topologies, which employ different control approaches and designs. Additionally, the harmonics caused by power converters change in time, and depend on numerous factors, including the interaction between inverter control, system impedance and background distortion [7].

On the contrary, harmonic studies for distribution systems, when performed, typically rely on simplified models, which may not be able to provide a realistic estimate of the harmonic phenomena observed in the grid. This simplification is due to different factors, including lack of detailed models and computational limits [8].

This paper is intended to fill in this gap, by proposing a new inverter model which allows studying in detail harmonic distortion caused by RESs in large distribution systems, while being relatively simple to deploy and keeping the simulation time limited.

The next section will summarize the main factors which determine harmonic interactions between RESs and the power grid, and which may result in amplification of certain harmonic components. Section III will describe the proposed model and will compare simulation results and computation time with existing models. Section IV and V will study mitigating solutions to control harmonic levels in a system operating under resonance conditions.

\section{HARMONIC INTERACTION BETWEEN RESS AND THE POWER GRID}

Harmonic levels caused by RESs depend on numerous factors: power converter topology, control system design, grid impedance and background harmonics are the main ones [9]. This section will briefly describe these factors to provide an overview of the problem under consideration and to describe the requirements for the development of a novel computer model. 


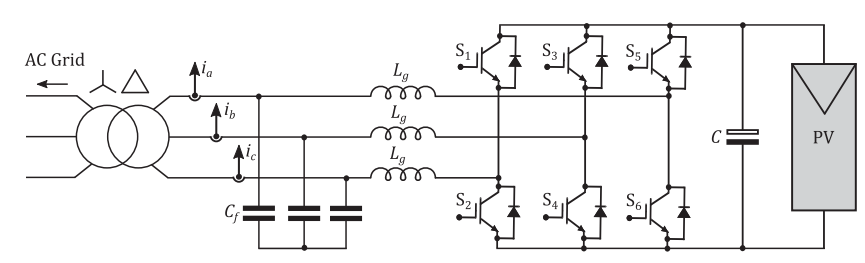

Fig. 1. Typical configuration of a PV system, including a 2-level three-phase VSC, an line filter, and a step-up transformer.

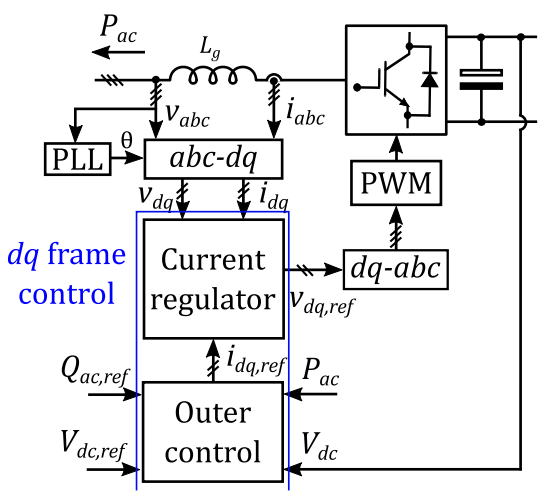

Fig. 2. Overview of the control blocks and of the measurements for the VSC shown in Fig. 1.

\section{A. RES Configuration and Control System}

In this paper, the topology shown Fig. 1 is considered, as it is very commonly deployed. A two-level three-phase voltagesource converter (VSC) is used to interface a photovoltaic (PV) panel to the grid; the grey box labelled 'PV' identifies the combination of the PV panel and of the dc-to-dc conversion stage, used to step-up the voltage across the dc-link capacitor $C$. The VSC consists of two solid-state switches per leg, controlled by using pulse width modulation (PWM) technique. A low-pass filters is employed on the ac side to attenuate the high-frequency components: in Fig. 1, the filter is a combination of the inductor $L_{g}$ and the capacitor $C_{g}$. A step-up transformer allows interfacing the PV system to the grid. A delta-connected transformer is used on the inverter side to block the injection of triplen harmonic components into the grid. The resistive components of the system are not shown in Fig. 1 for simplicity, but they are included in the computer model.

Fig. 2 shows the main blocks of the control diagram used to regulate the VSC operation. While this paper only provides an overview of the typical control system configuration, more details can be found in [11]-[13]:

- The outer control loop calculates the $d q$ reference currents $i_{d q, \text { ref }}$ (zero sequence components are not considered in this work as the system is symmetrical) [14]. The $q$-axis reference current $i_{q, r e f}$ is obtained from the reactive power reference value $Q_{a c, r e f}$. In most cases, the power factor is unity and therefore the $Q_{a c, \text { ref }}=0$, but in principle the power factor can be set to any values between 0 and 1 . The $d$-axis reference current is obtained from the dc-link voltage controller.

- The currents $i_{q d, r e f}$ are fed to the current regulator, where they are compared to the measured currents $i_{d q}$. The error

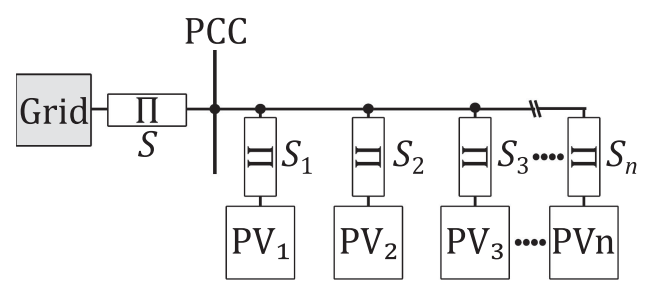

Fig. 3. Single-line diagram of the distribution system used for the analysis: each PV unit is connected to the PCC by means of pi-sections $\left(S_{1}\right.$ to $\left.S_{n}\right)$. The grid equivalent includes a voltage source and a Thevenin impedance, and it is connected to the PCC by cable section $S$.

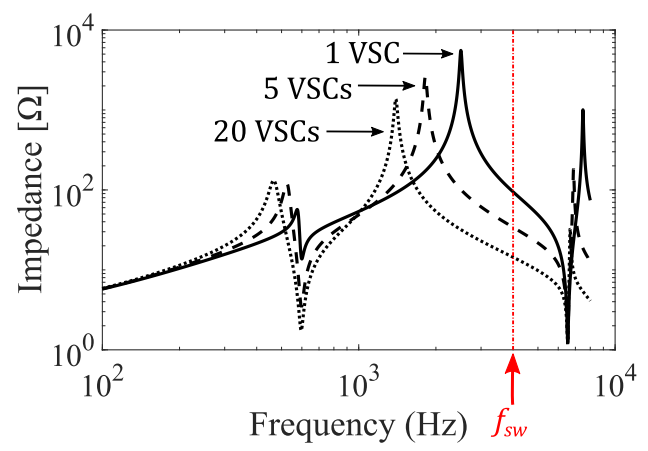

Fig. 4. Impedance plots for the distribution system shown in Fig. 3, with varying number of VSCs connected to the PCC.

term is fed into a proportional-integral (PI) block. The output of the current regulator are the reference voltages $v_{q d, \text { ref }}$.

- The reference voltages $v_{q d, r e f}$ are compared with the carrier wave in the PWM block, and the switching pulses for the inverter are generated.

While this paper focuses on the system shown in Fig. 1, it is possible to extend the analysis carried out to other RESs interfaced to the grid through a VSC, as for the purpose of harmonic interaction, the dc side converter dynamics can be ignored [15].

\section{B. Harmonic Impedance}

Connecting a large number of RESs in close proximity has an impact on the grid impedance, although the effect of each device may be small.

The distribution system shown in Fig. 3 is used to study this phenomenon. A variable number of PV units with the same configuration as the one shown in Fig. 1 is connected to the point of common coupling (PCC) by means of cable sections $S_{1}$ to $S_{n}$, and PCC is connected to the rest of the grid through an equivalent line $S$. The system parameters are provided in Appendix A. The cables and the equivalent lines are represented by pi-sections because including the capacitance in the system model is important for harmonic studies [9].

The system harmonic impedance measured at the PCC with a varying number of VSCs is shown in Fig. 4: the peaks and the valleys correspond to parallel and series resonances, respectively. With an increasing number of VSCs in service, the resonance points shift towards lower frequencies. This can be explained by observing that each VSC and filter combination 
causes an increase in the total reactance of the system, thus lowering the resonant frequency, which in the simplest form can be expressed as $f_{r e s}=1 / 2 \pi \sqrt{L C}$. Therefore, varying the number of VSCs connected on the system has an impact on the total impedance [16]. The impedance plot is not shown for higher frequencies because the validity of the computer models used in this work is limited to the lower frequency range. Different models are required to study higher frequencies as described in [10]. The switching frequency $f_{s w}$ is also indicated on the same graph to provide a comparison with the grid resonant frequencies.

The dependency of the system impedance on the number of VSCs in service contributes to the variability of harmonic levels in systems with a large number of RESs, as harmonic current and voltage components are amplified when a resonance condition exists at the same frequency. Specifically, harmonic current components are amplified by parallel resonances (which correspond to peaks in the impedance scan), while voltage harmonic components are amplified by series resonance (dips in the impedance scan) [9].

Therefore, harmonic levels in systems with a varying number of VSCs are determined by the harmonic injection of each unit, but also by the resonance conditions obtained with a varying number of units in service.

\section{Background Distortion}

Due to the presence of non-linear loads and sources, the grid voltage includes time-varying harmonic components, which are often referred to as 'background distortion'. While the devices that generate harmonics are dispersed in the grid, it is customary to model background distortion with harmonic voltage sources connected in series to the Thevenin equivalent voltage at fundamental frequency.

Background distortion is fed into the inverter control through current and voltage measurements, as shown in Fig. 2. As a result, the inverter generates additional harmonic components when compared to the case of sinusoidal voltage source, due to the frequency response of the VSC control loop. The ratio between harmonic currents and voltages at the same frequency is referred to as 'auto-sensitivity' in [6], while the ratio between harmonic currents and voltages at different orders is referred to as 'cross-sensitivity'. In [6], the auto- and cross-sensitivities for different inverter topologies are compared, and it is shown that there is a large variability due to the control system response employed in each case.

\section{Harmonic Interaction for the Studied System}

The impact of the factors described above is illustrated by means of a case study, that is based on the system shown in Fig. 3. Five PVs are considered, and each one employs the control scheme illustrated in Fig. 2. The grid equivalent voltage source includes the following components in addition to the fundamental: $5 \%$ amplitude for the $5^{\text {th }}$ harmonic, and $4 \%$ amplitude for the $7^{\text {th }}$ and $11^{\text {th }}$ harmonics.

Fig. 5 shows the PCC voltage spectrum for two cases: 1) no VSCs in service (green bars), and 2) five VSCs operating at full power (black bars). For the case with no VSCs, the voltage components observed at the PCC are very similar to the ones

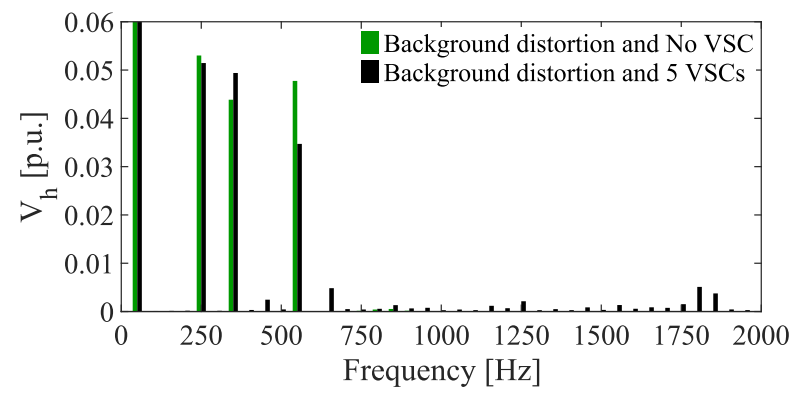

Fig. 5. Frequency spectrum of voltage at the PCC for two cases: with no VSC in service and with 5 VSCs in service. Background distortion is included in both cases.

included in the background distortion, although the voltage amplitudes are slightly amplified due to the frequency response of the grid.

When five VSCs are in service, the $5^{\text {th }}$ component is slightly damped, while the $7^{\text {th }}$ component increases. However, the impact on low-order harmonics is overall minimal. The most interesting phenomena appear with increasing frequencies: the $11^{\text {th }}$ component is significantly damped, while new harmonic components appear. The most noticeable are the $13^{\text {th }}$ harmonic and components with frequencies around $1800 \mathrm{~Hz}$ ( $36^{\text {th }}$ harmonic). Even if the amplitude of the high-frequency terms are small, they exceed the limits included in some grid codes, such as [4], [5]. The source of these harmonic components will be discussed in Section III-C.

The results shown in Fig. 5 indicate that the interaction between grid impedance, background distortion and VSC control system response is complex: even a small number of VSCs may lead to generation of new harmonic components which are not present in the background voltage distortion, thus illustrating the concept off cross-sensitivity introduced in Section II-C.

\section{VSC MODELING FOR HARMONIC STUdIES}

The summary provided in the previous section illustrates that, when numerous VSCs are connected in close proximity, the harmonic analysis is complex due to the presence of multiple interacting factors.

In order to study the impact of VSCs on harmonic levels, several examples of VSC models have been proposed for harmonic studies [8], which can be categorised in two main groups: frequency-domain models [15], [17] and time-domain models [18], [19] . Each approach has its own advantages and limitations.

Frequency-domain models represent the VSC, the grid impedance and the current injection at each harmonic through matrices and vectors. Once the model is solved in the frequency domain, the voltage and current harmonic components are combined by using the reverse Fourier Transformation, and the time-domain waveforms are reconstructed. The main advantage of frequency-domain models is the small simulation time. However, they are characterized by a few disadvantages: the accuracy of the time-domain waveforms depends on the resolution of the Fourier Transformation, and the frequency-domain system model is time-consuming to develop when a large grid is 
considered. In particular, if different grid configurations need to be analyzed, a separate matrix needs to be build for each case.

Time-domain models are more intuitive to use, as each component is represented by using a graphical user interface. This approach allows studying multiple configurations using the same model, as different components can be easily switched on and off, even within the same simulation. One of the main limitations of time-domain model is that they typically require long simulation times, in particular when a large number of VSCs is included.

Based on the comparison above, while it is recognized that frequency-domain models are advantageous for certain applications, this paper will focus on the development of an effective and simple time-domain model to be used in large distribution systems.

\section{A. VSC Modeling in Time Domain}

Traditionally, system level harmonic studies are performed through current injection methods where the VSC is represented by a number of fixed and parallel-connected current sources, with or without parallel admittance. This approach is known as the Norton equivalent model [16], [21]. Although this method significantly reduces the simulation time, it exhibits a poor accuracy, as it neglects the interactions described in Section II, and the consequent time-variation of harmonic components.

On the other hand, Detailed Models (DMs), while representing accurately the VSC harmonic injection, result in excessively long simulation times, in particular when a large number of VSCs is included [8].

In order to address the drawbacks of the two approaches described above, alternative modelling techniques have been proposed. In [20], the power switches are represented as controllable resistors, that are switched on and off based on the gate signal. The value of the resistance is determined by the valve current/voltage relationship. Since these models neglect the transient state of solid-state switches, the simulation time is reduced by at least $50 \%$ when compared to the DM. However, the simulation time cannot be further reduced as the number of electrical nodes to solve remains the same as in the DM.

Average Models (AMs) been proposed to achieve a more significant reduction of the simulation time [21]. Fig. 6(a) illustrates the single phase AM for the PV system shown in Fig. 1: the VSC is replaced by a controlled voltage source per phase $\left(V_{x n}\right.$, with $\left.x=[a, b, c]\right)$ on the ac side, and a controlled current source $\left(i_{d c}\right)$ on the dc side. AMs offer fast simulation speed, but they do not give any insights on harmonic and inter-harmonic interactions associated with the VSC, since they do not include a representation of the power switches.

To overcome the shortcomings of AMs, a switching AM (SAM) incorporating the VSC switching dynamics is introduced in [22]. The SAM uses controllable voltage and current sources to represent the active elements of the converter, and the harmonic content is generated using two switching functions. This model supports all modulation techniques. Fig. 6(b) illustrates the single-phase SAM for the system shown in Fig. 1: the two switching function blocks $f_{1}(\mathrm{t})$ and $f_{2}(\mathrm{t})$ are introduced to mimic the converter's ac and dc side switching dynamics,

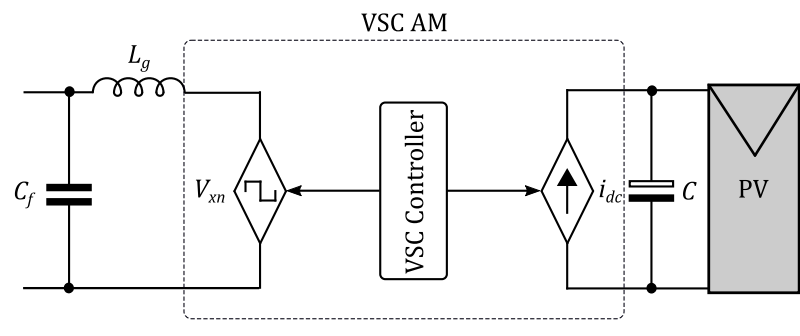

(a)

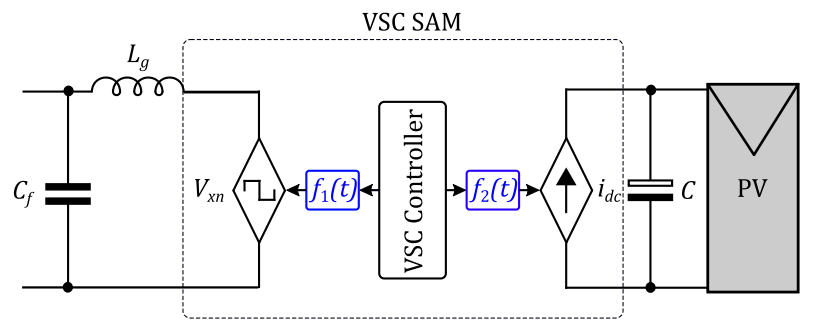

(b)

Fig. 6. Single-phase equivalent models for the system shown in Fig. 1: (a) Average model (AM) and (b) Switching Average Model (SAM). The first model consists of controlled current and voltage sources. The second model is obtained by adding two switching functions to the conventional average model. In both figures, the label $V_{x n}$ refers to the line-to-neutral voltage for each phase $x=a, b, c$.

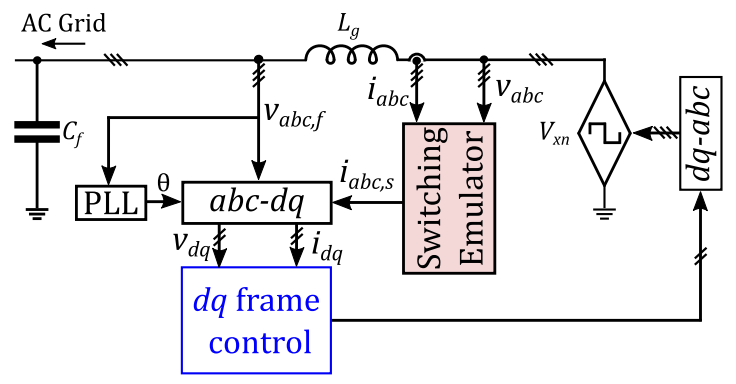

Fig. 7. Three-phase equivalent diagram of the proposed model, that includes a switching emulator and is referred to as AMSE. Only one controlled voltage source is shown to simplify the figure.

respectively. Although the SAM improves simulation accuracy, it still exhibits a high computation time in presence of a large distribution grid.

In the next section, a novel reduced order module is formulated, to decrease the computation time while maintaining simulation accuracy.

\section{B. Average Model With Switching Emulator}

Fig. 7 shows the three-phase equivalent diagram of the proposed time-domain model. Similarly to the AM, this approach employs a controllable voltage source to represent the active elements of the converter. A switching emulator is introduced to mimic the converter modulation - the resulting model is therefore referred to 'average model with switching emulator', or AMSE. The ' $d q$ frame control' block is the same shown in Fig. 1.

In Fig. 7, the average line-to-neutral voltage, $V_{x n}$, is expressed by the following equation:

$$
V_{x n}=\frac{1}{2} d_{x} V_{d c}
$$




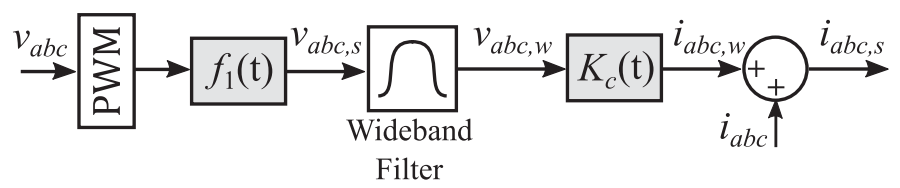

Fig. 8. Detailed representation of the switching emulator shown in Fig. 7.

where $V_{d c}$ is the dc link voltage and $d_{x}$ is the phase modulation index. The phase modulation index, $d_{x}$, is obtained from the following equation [21]:

$$
d_{x}=m_{x} \cos \left(\omega t+\delta+p_{x}\right)
$$

where $m_{x}$ is the modulation index, $\delta$ is the phase angle of the converter terminal voltage with respect to the voltage at the PCC and $p_{x}$ is the phase shift. For a balanced three-phase system, $m_{a}=m_{b}=m_{c}$, and the phase shifts are equal to $0,-2 \pi / 3$ and $-4 \pi / 3$ for phase $a, b$ and $c$ respectively.

Fig. 8 shows the schematic diagram of the switching emulator: it consists of a switching function block $f_{1}(\mathrm{t})$, a wideband filter and a variable gain $K_{c}(\mathrm{t})$. The function $f_{1}(\mathrm{t})$ is the same employed in the AM, and shown in Fig. 6(a).

The normalised valve side voltage $v_{a b c}$ is fed through a PWM block and the function block $f_{1}(\mathrm{t})$ to generate a pulsed valve side voltage $v_{a b c, s}$. For a two-level VSC, the phase voltage $v_{x, s}$ is calculated from the switching function $S_{f x}$, according to the following expressions:

$$
\begin{aligned}
v_{x, s} & =\frac{1}{2} S_{f x} V_{d c} \\
S_{f x} & =S_{f u}-S_{f l}
\end{aligned}
$$

where $S_{f u}$ and $S_{f l}$ represent the states of upper and lower legs valves, $S_{u}$ and $S_{l}(u=[1,3,5], l=[2,4,6])$ respectively. The values of the switching function, $S_{f x}$, are limited to \pm 1 , and are obtained from the PWM block.

The converted signal $v_{a b c, s}$ is fed through a wideband filter. This filter is tuned to pass frequencies between the cut-off frequency of the ac filter (the combination of $L_{g}$ and $C_{g}$ in Fig. 1) and the converter switching frequency $f_{s w}$. The output of the wideband filter is fed through the gain block to obtain a current signal. The gain $K_{c}(\mathrm{t})$ is adjusted in real-time based on converter output power. The current signal $i_{a b c, w}$ is added to the measured valve side ac current $i_{a b c}$. The resulting current, $i_{a b c, s}$, is used as an input to the inner current loop, shown in Fig. 2.

As a result of the steps shown in Fig. 8, the phase current in the AMSE is expressed as follows:

$$
i_{x s}=\frac{1}{2} S_{f x} V_{d c} \cdot \frac{2 \zeta \omega_{c} s}{s^{2}+2 s \zeta \omega_{c}+\omega_{c}^{2}} K_{c}
$$

where $\omega_{c}$ is the center frequency and $\zeta$ is the damping ratio of the wideband filter.

\section{Numerical Analysis}

Fig. 9 compares the voltage profile at the PCC for the system studied in Section II-B, which includes five VSCs operating at full power. In this case, the grid voltage is sinusoidal at fundamental frequency. The voltage profiles are obtained using three different modeling techniques: DM, SAM and AMSE.

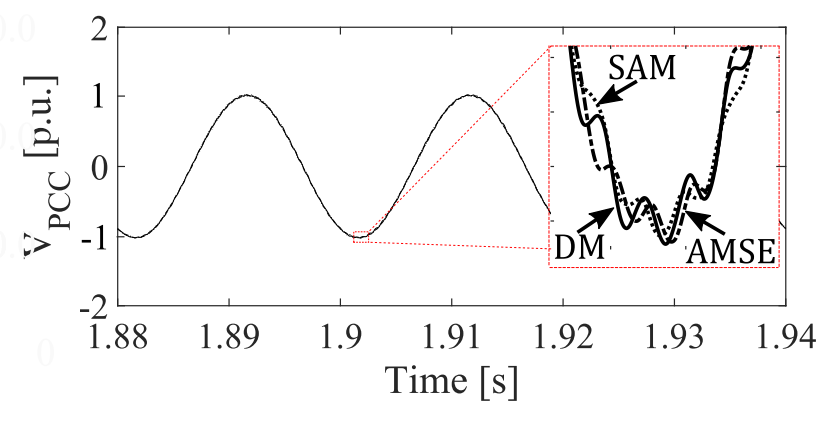

Fig. 9. Voltage at the PCC for the case of 5 VSCs in service and sinusoidal grid voltage. Three models are compares: Switching Average Model (SAM), Detailed model (DM) and Average Model with Switching Emulator (AMSE).

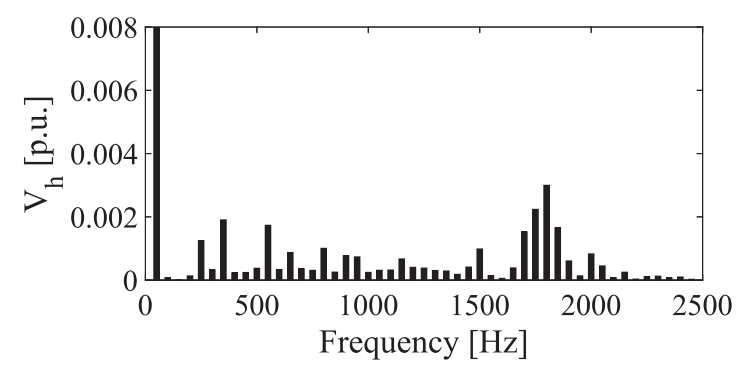

(a) SAM

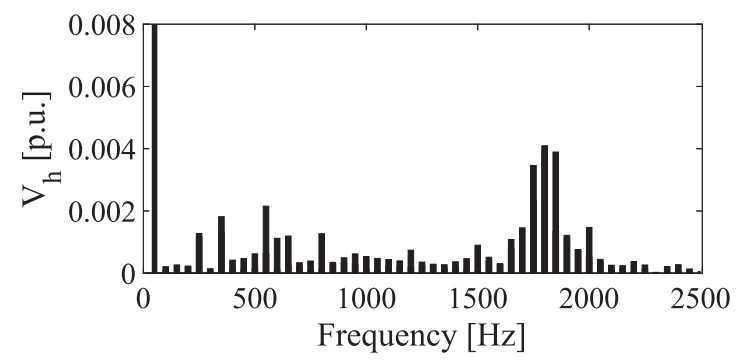

(b) $\mathrm{DM}$

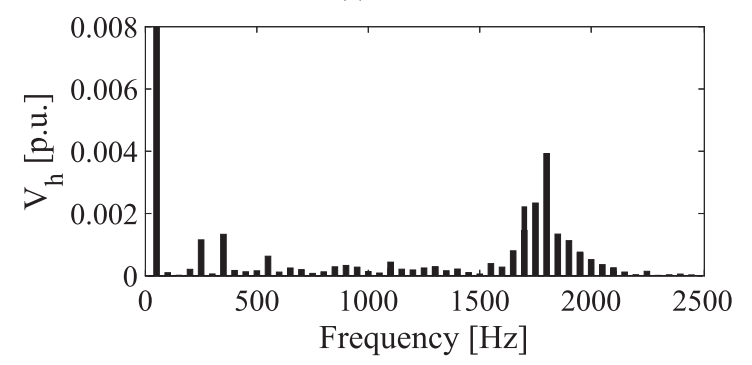

(c) AMSE

Fig. 10. Frequency spectrum of the voltage at the PCC for the three cases shown in Fig. 9: (a) Switching Average Model, (b) Detailed Model and (c) Average Model with Switching Emulator.

The waveforms included in Fig. 9 show that both the SAM and the AMSE closely follow the detailed model even for small oscillations. The results obtained with the AM are not included because this modeling approach is only able to produce a sinusoidal waveform at fundamental frequency.

Fig. 10 illustrates the frequency spectrum for each one of the waveforms shown in Fig. 9, to provide insights on the harmonic content obtained by using each model. The $y$-axis scale is small as in this case no background distortion is present and the only harmonics observed are the ones due to VSC operation. The $x$-axis is limited to $2500 \mathrm{~Hz}$ because above this frequency no 
TABLE I

COMPUTATION TIME FOR DIFFERENT MODELS

\begin{tabular}{cccc}
\hline Modeling type & DM & SAM & AMSE \\
\hline Computation time & $27 \mathrm{~s}$ & $9.5 \mathrm{~s}$ & $4.5 \mathrm{~s}$ \\
\hline Simulation step & \multicolumn{3}{c}{$5 \mu \mathrm{s}$} \\
\hline \multirow{2}{*}{ Simulation Platform } & \multicolumn{3}{c}{ Simulink/SimPowerSystems } \\
& on a $2.6 \mathrm{GHz}$ core-i7 4960 pc with 16 GB ram \\
\hline
\end{tabular}

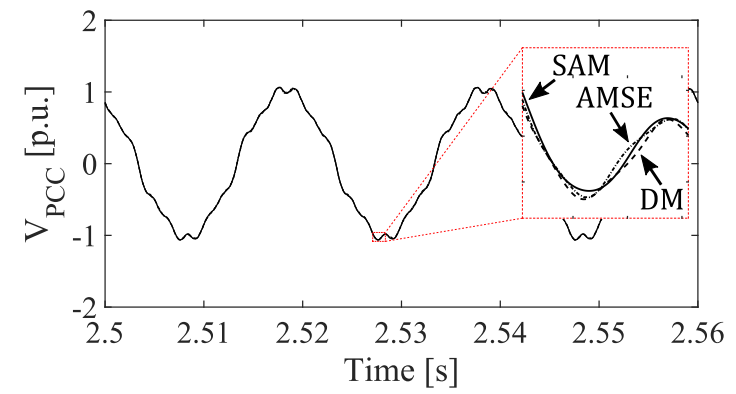

Fig. 11. Voltage at the PCC for the case of 5 VSCs in service and background distortion included in the grid voltage source. Three models are compared: SAM, DM, and AMSE.

significant harmonic components are observed. In particular, the switching component at $4 \mathrm{kHz}$ is effectively damped by the inverter output filter. The results displayed in Fig. 9 show that both the AMSE and the SAM show a good accuracy compared to the DM, although the harmonic components obtained with the AMSE are slightly damped compared to the DM.

The spectra included in Fig. 10 show harmonic components in the region around $1800 \mathrm{~Hz}$, which are due to harmonic interaction between the VSCs and the grid impedance. To understand this phenomenon, one has to consider the frequency scan shown in Fig. 4: when five VSCs are in service, a resonance peak can be identified just below $2 \mathrm{kHz}$. A zoomed-in version of the graph shows that this peak is obtained for a frequency close to $1800 \mathrm{~Hz}$. As a results, the control system response of the five VSCs tends to amplify harmonic components in this frequency range. With varying number of VSCs in service, the frequency of the oscillations changes, as it is dependent on the frequency of the resonance peaks of the grid impedance. Even if the amplitude of the oscillations is quite small, an accurate model for harmonic studies must be able to duplicate these phenomena.

Together with accuracy, computation time is a second essential criterion to consider for a model used to analyze power quality performance of inverters. Table I summarizes the computation time of each modeling technique. The AMSE features the smallest computation time, while still allowing great accuracy as shown in Fig. 10: for the case of five VSCs in service, the simulation time is six times less than the one required by the DM, and approximately half than the one required by the SAM. For simulations including a larger number of VSCs, the advantage of the AMSE in terms of computation time becomes more evident.

The performance of AMSE is further investigated by including harmonic components in the grid equivalent source. Figs. 11 and 12 show the time domain representation and frequency spectrum of the voltage at PCC, respectively. The results are compared for the same modeling techniques discussed earlier: DM,

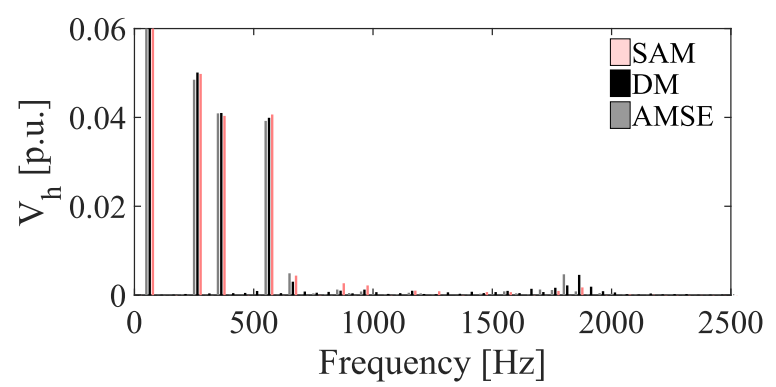

Fig. 12. Frequency spectrum of voltage at the PCC for the waveforms shown in Fig. 11.

SAM and AMSE. In this case, the distortion is more visible because background harmonics are included, an they dominate the frequency spectrum at low frequency. As a result, the three models show a good agreement for frequencies below $1 \mathrm{kHz}$. For higher frequencies, the SAM is less sensitive towards the resonant components (between 1750 and $1850 \mathrm{~Hz}$ ).

The results analysed in this section allow concluding that the proposed model is accurate in reproducing harmonic distortion caused by VSCs, both in presence of sinusoidal voltage source and of harmonic distortion. At the same time, the AMSE exhibits a low simulation time compared to both the DM and the SAM.

\section{HARMONIC MitigATION}

Different mitigating solutions are available to reduce the effect of harmonics. The most effective approach consists in reducing harmonic current emissions from the source, i.e. the VSCs, and standards such as [4] and [5] have been developed with this purpose. As a result, the latest generations of VSCs and non-linear loads generate small amounts of harmonics, as illustrated by the waveforms shown in Fig. 9. However, with increasing levels of non-linear loads and RESs, and interaction with the grid impedance, it is expected that harmonic levels may be exceeded under certain operating conditions.

Passive filters are used to mitigate excessive harmonics by creating a low-impedance path at one or more frequencies [9]. While passive filters are a proven technology with wellestablished manufacturing and design practice, there is a concern that they may not be suitable to mitigate harmonic problems in power systems with high level of RESs, due to a few reasons:

1 RESs generate harmonics across a broad range of frequencies which changes in time, while passive filters are tuned to specific frequencies. Therefore, they may not be effective under all operating conditions.

2 With growing number of harmonic sources, the duties imposed on the filters will increase, thus causing overload conditions which may lead to filter failure [23].

3 The combination of the filter impedance and a changing numbers of VSCs may introduce new resonance conditions in the system.

Given the disadvantages highlighted above, this paper proposes the use of Active Filters (AFs) to mitigate harmonic components in systems with high penetration of RESs.

The concept of AF has been proposed a few decades ago in [24] and consists in using a shunt-connected three-phase power converter to absorb harmonic current components from 


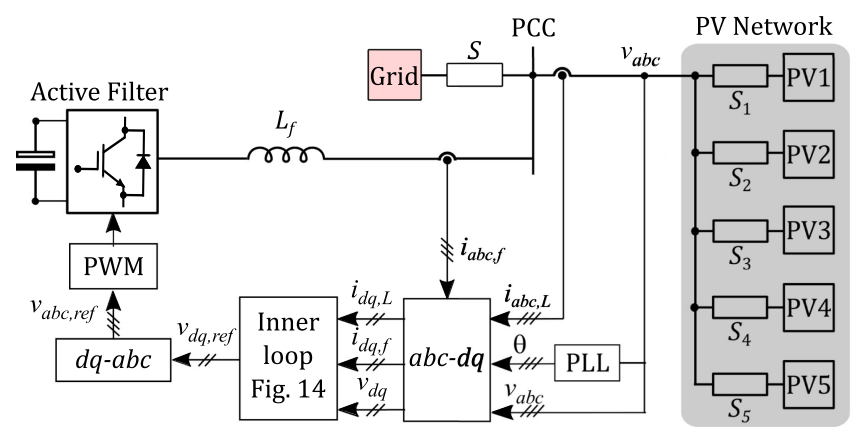

Fig. 13. The modified system where an AF is connected at the PCC. The main control blocks and measurements are included.

the grid. More recently, there has been a renewed interest in AFs, due to a few reasons, including: increasing levels of harmonics, improved performance of power converters, and a reduction in the cost of these devices [25]-[28] .

Using this alternative solution alleviates some of the concerns highlighted above for passive filters:

1 AFs allow mitigating a broad spectrum of harmonic components, and the control system quickly responds to changes in the frequency of harmonic currents to compensate.

2 It is less likely to overload and damage an AF due to the protection installed both at the control level and at the relay level.

3 An AF does not alter the grid frequency response to the same extent as a passive filter. Some interaction still exists, due to the coupling of the power converter capacitance to the system through power switches operation.

For the system under study, an AF is installed at the PCC as shown in Fig. 13, together with the main control blocks. The operating principle of the AF is the following: the power converter is modulated in such a way to inject harmonic currents which are equal in magnitude and opposite in phase with respect to the harmonic currents detected in the power grid.

To achieve this objective, a $d q$ frame-based control is implemented. The grid voltages $v_{a b c}$ are fed through a Phase-LockedLoop (PLL), which detects the phase $\theta$ with respect to phase $a$ grid voltage, that is one of the inputs for the block that performs the $d q$ transformation [14]. The voltages $v_{a b c}$, the AF output currents $i_{a b c, f}$ and the line currents $i_{a b c, L}$ are transformed in the equivalent $d q$ domain. These quantities are then the input to the inner loop, which will be described in the next paragraph. The inner loop generates the reference voltages $v_{d q, \text { ref }}$, that are then transformed in the $a b c$ domain and used to generate the switching pulses.

Fig. 14 shows the inner current loop, for both $d$ and $q$ current components. Only the $d$-axis path is described as $d$ and $q$ are almost identical. Since the $d q$ axes rotates at the same speed as the grid voltage phasors, the fundamental current correspond to a dc component in the equivalent domain [29]. Therefore, it can be removed easily by using a dc filter, without affecting the oscillatory components which are obtained from the harmonic currents. The output of the filter is the harmonic reference current. This current is compared with the measured current at AF terminals. The error term is fed to the PI controller, and the

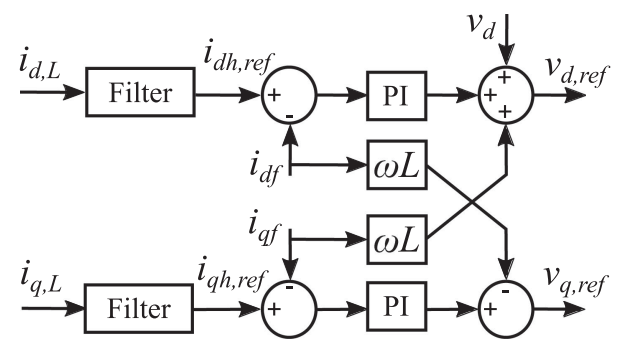

Fig. 14. Details of the Inner Loop: the reference voltages $v_{d, \text { ref }}$ and $v_{q, \text { ref }}$ are obtained from the harmonic reference currents and from the voltages measured at the PCC.

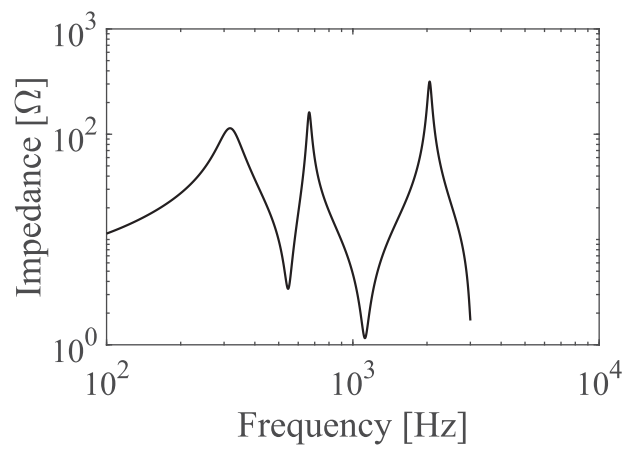

Fig. 15. Impedance plot for the modified distribution system: low resonance peaks appear in proximity of the $7^{\text {th }}$ and $11^{\text {th }}$ harmonic

output is the reference harmonic voltage. This voltage is added to the measured grid voltage $v_{d}$, to provide the reference voltage $v_{d q, r e f}$, that are used to generated the switching pulses.

\section{Simulation Results}

This section presents simulation results showing the effectiveness of the proposed AMSE under resonant conditions, and the use of AF to mitigate harmonic amplification.

The system configuration used in the previous sections is adopted (five VSCs are in service), and the AF is connected at the PCC, as shown in Fig. 13. The cable lengths are modified to create two resonance points close to the $7^{\text {th }}$ and $11^{\text {th }}$ harmonic. The new cable lengths are listed in Appendix A. The impedance plot for the modified system is provided in Fig. 15: this graph shows a few (series and parallel) resonance conditions up to $3 \mathrm{kHz}$.

Using this new system topology, two cases will be analyzed in the next sections. For both cases, current waveforms are plotted as this quantity is the one affected the most by AF operation.

\section{A. Sinusoidal Voltage Source}

The current at the PCC is shown in Fig. 16, where the AMSE is compared with the detailed model, that provides the benchmark for accuracy. With the updated cable lengths, new resonance conditions are met at the lower frequencies, and therefore the current at the PCC results visibly distorted. The results in Fig. 16 indicate that the AMSE is able to track these oscillations, thus confirming that the proposed model is accurate. Fig. 17 shows the same simulation when the AF is implemented: the improvement of the waveform is quite significant and demonstrates that 


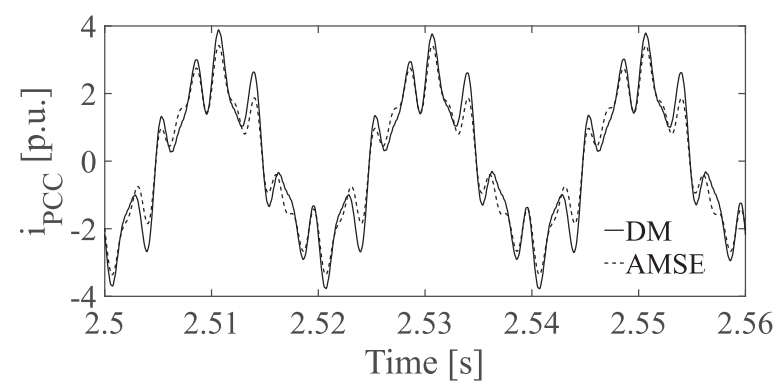

Fig. 16. Current at the PCC for the modified distribution system, with five VSCs in service and no background distortion.

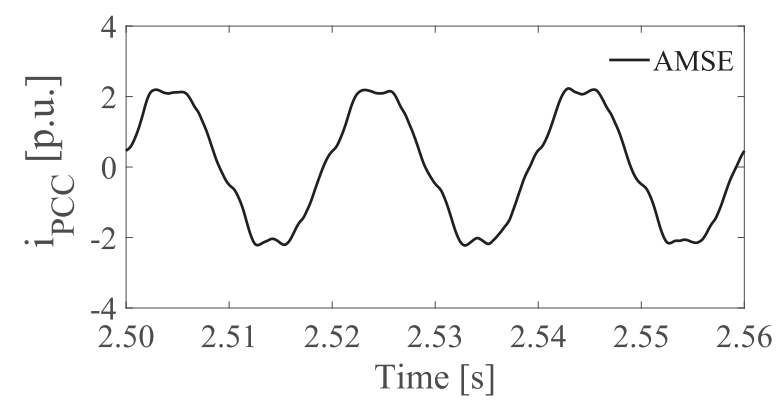

Fig. 17. Current at the PCC for the same case as in Fig. 16, with AF in service.

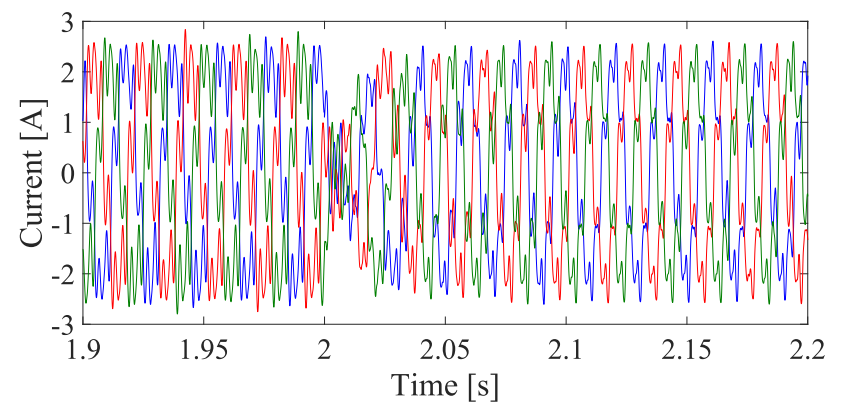

Fig. 18. Current at the PCC for the modified system, 5 VSCs in service, and with background distortion included. The AF enters in service at $t=2 \mathrm{~s}$.

the $\mathrm{AF}$ is effective in removing the harmonic currents generated by the VSCs.

\section{B. Background Distortion}

The case studied in the previous section is repeated with background distortion is included into the model, using the same spectrum applied in Section II and Section III. The current waveforms obtained with the AMSE are shown in Fig. 18, where the $\mathrm{AF}$ is activated at $t=2 \mathrm{~s}$. The $\mathrm{AF}$ is not as effective in mitigating the harmonic components in this case, because the majority of the distortion is caused by the grid, which acts as an infinite source of harmonics. The current spectra shown in Fig. 19 provide some further insights and indicate that the distortion is mostly caused by the harmonic components included in the grid voltage. The AF mitigates the harmonic components caused by the VSCs. This case illustrates the importance of controlling voltage distortion in the grid, because pre-existing harmonics are difficult, if not impossible

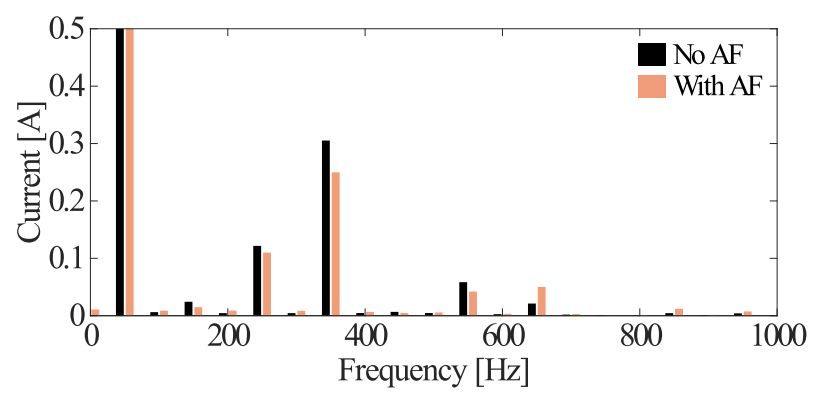

Fig. 19. Current harmonic components for the case shown in Fig. 18: comparison of harmonic components for $t<2 \mathrm{~s}$ (No AF) and for $t \geq 2 \mathrm{~s}$ is shown.

to mitigate. Very large filters (either passive or active) would be required, but their cost and size would be prohibitive.

\section{CONCLUSIONS}

This paper focused on the modeling of harmonic distortion caused by RESs in large distribution systems: after a summary of the main factors which affect harmonic levels in the grid, a new model for harmonic studies is proposed, referred to as 'average model with switching emulator' (AMSE).

Comparison between the AMSE and existing models has been carried out for a distribution system including multiple VSCs, and the impact of resonance condition and background distortion has been analysed. The results allow concluding that the proposed model accurately replicates harmonic distortion, while significantly reducing the simulation time.

The last section of the paper studies harmonic levels in a system operating under severe resonance conditions. The results indicate that the proposed model is effective in reproducing harmonic amplification under these conditions as well. It also shows the effectiveness of the $\mathrm{AF}$ in mitigating harmonic currents generated by the VSCs, while background distortion is mostly unaffected.

The next steps of this analysis will address the impact of both RESs and AFs on the higher order harmonics (known as supraharmonics [30]), as both these categories of devices implement high switching frequencies. The study of these frequencies require a different type of modeling and therefore it cannot be addressed using the approach described in this paper.

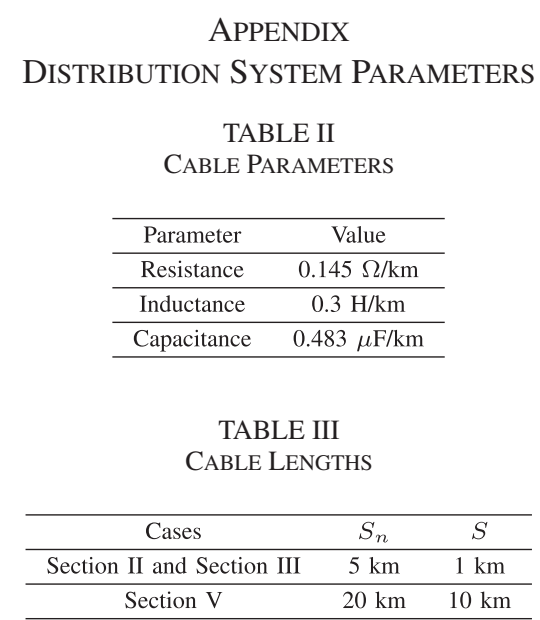


TABLE IV

PV SYSTEM MAIN PARAMETERS

\begin{tabular}{ccc}
\hline Devices & Specification & Rating / Value \\
\hline \multirow{3}{*}{ VSC } & Rated power & $2 \mathrm{~kW}$ \\
& Rated ac voltage & $140 \mathrm{~V}$ \\
& Rated dc voltage & $250 \mathrm{~V}$ \\
\hline ac inductor & $L_{g}$ & $2.2 \mathrm{mH}$ \\
\hline ac capacitor & $C_{f}$ & $10 \mu \mathrm{F}$ \\
\hline dc link capacitor & $C$ & $1020 \mu \mathrm{F}$ \\
\hline
\end{tabular}

\section{REFERENCES}

[1] O. S. Nduka and B. C. Pal, "Harmonic domain modeling of PV system for the assessment of grid integration impact," IEEE Trans. Sustain. Energy, vol. 8, no. 3, pp. 1154-1165, Jul. 2017.

[2] J. F. G. Cobben, "Power quality implications at the point of connection," Ph.D. thesis, Dept. Elect. Eng., Eindhoven Univ. Technol., Eindhoven, the Netherlands, 2007.

[3] IEEE Standard for Interconnection and Interoperability of Distributed Energy Resources With Associated Electric Power Systems Interfaces, IEEE Standards Coordinating Committee 21, 1547-2018.

[4] IEEE Recommended Practice and Requirements for Harmonic Control in Electric Power Systems, IEEE Standard 519-2014.

[5] Electromagnetic Compatibility (EMC) Part 2-2: Environment Compatibility Levels for Low-Frequency Conducted Disturbances and Signalling in Public Low-Voltage Power Supply Systems, 2.1 ed., IEC Standard 61000-2-2, Jun. 1, 2017.

[6] "Power quality aspects of solar power," CIGRE working Group JWG C4/C6.29, Technical Brochure 672, Paris, France, Dec. 2016.

[7] H. Hu et al., "Potential harmonic resonance impacts of PV inverter filters on distribution systems," IEEE Trans. Sustain. Energy, vol. 6, no. 1, pp. 151-161, Jan. 2015.

[8] MIGRATE Project, "Simulation models for power-quality studies in power-electronics rich power networks," Tech. Rep. 5.2, Jun. 2017. [Online]. Available: https://www.h2020-migrate.eu/downloads.html

[9] J. Arrilaga and N. R. Watson, Power System Harmonics, 2nd ed., Hoboken, NJ, USA: Wiley, 2003.

[10] J. A. Martinez-Velasco, Power System Transients: Parameter Determination. Boca Raton, FL, USA: CRC Press, 2009.

[11] R. Belaidi, M. Fathi, M. M. Larafi, G. M. Kaci, and A. Haddouche, "Power quality improvement based on shunt active power filter connected to a photovoltaic array," in Proc. IEEE Renewable Sustain. Energy Conf., 2015, pp. 1-6.

[12] T. Geury, S. Pinto, and J. Gyselinck, "Three-phase power controlled PV current source inverter with incorporate active power filtering," in Proc. Annu. Conf. IEEE Ind. Electron. Soc., 2013, pp 1374-1379.

[13] A. S. Shirbhate and S. D. Jawale, "Power quality improvement in PV grid connected system by using active filter," in Proc. Int. Conf. Energy Efficient Technol. Sustain., 2016, pp 388-395.

[14] R. H. Park, "Two reaction theory of synchronous machines," AIEE Trans., vol. 48, pp. 716-730, 1929.

[15] J. Kwon et al., "Harmonic interaction analysis in grid connected converter using harmonic state space (HSS) modeling," IEEE Trans. Power Electron., vol. 32, no. 9, 6823-6835, Sep. 2017.

[16] Task Force on Harmonics and Simulations, "Modeling and simulation of the propagation of harmonics in electric power networks.I. Concepts, models and simulation techniques," IEEE Trans. Pow. Del., vol 11, no. 1, pp. 452-465, Jan. 1996.

[17] J. Lyu, X. Zhang, X. Cai, and M. Molinas, "Harmonic state-space based small-signal impedance modeling of modular multilevel converter with consideration of internal harmonic dynamics," IEEE Trans. Power Electron., vol. 34, no. 3, pp. 2134-2148, Mar. 2019.

[18] Y. Gu, N. Bottrell, and T. C. Green, "Reduced-order models for representing converters in power system studies," IEEE Trans. Power Electron., vol. 33, no. 4, pp. 3644-3654, Apr. 2018.

[19] W. Lin and D. Jovcic, "Average modelling of medium frequency dc-dc converters in dynamic studies," IEEE Trans. Power Del., vol. 30, no. 1, pp. 281-289, Feb. 2015.

[20] U. N. Gnanarathna, A. M. Gole, and R. P. Jayasinghe, "Efficient modeling of modular multilevel HVDC converters (MMC) on electromagnetic transient simulation programs," IEEE Trans. Power Del., vol. 26, no. 1, pp. 316-324, Jan. 2011.
[21] J. Peralta-Rodriguez, "Dynamic averaged models of VSC-based HVDC systems for electromagnetic transient programs," Ph.D. thesis, Dept. Elect. Eng., Universite de Montreal, Montreal, QC, Canada, 2013.

[22] J. Peralta et al., "Detailed and averaged models for a 401-level MMCHVDC system," IEEE Trans. Power Del., vol. 27, no. 3, pp. 1501-1508, Jul. 2012

[23] "Guide to the specification and design evaluation of AC filters for HVDC projects," CIGRE Technical Brochure 139, Paris, France, 1999.

[24] H. Akagi, Y. Kanazawa, and A. Nabae, "Instantaneous reactive power compensators comprising switching devices without energy storage components," IEEE Trans. Ind. Appl., vol. IA-20, no. 3, pp. 625-630, May 1984.

[25] J. Nastran, R. Cajhen, M. Seliger, and P. Jereb, "Active power filter for nonlinear AC loads," IEEE Trans. Power Electron., vol. 9, no. 1, pp. 92-96, Jan. 1994.

[26] B. Singh, K. Al-Haddad, and A. Chandra, "A review of active filters for power quality improvement," IEEE Trans. Ind. Electron., vol. 46, no. 5, pp. 960-971, Oct. 1999.

[27] L. Asiminoaei, F. Blaabjerg, and S. Hansen, "Evaluation of harmonic detection methods for active power filter applications," in Proc. 20th Аnпu. IEEE Appl. Power Electron. Conf. Expo., Mar. 2005, pp. 635-641.

[28] G. Superti-Furga and G. Todeschini, "Discussion on instantaneous $p-q$ strategies for control of active filters," IEEE Trans. Power Electron., vol. 23, no. 4, pp. 1945-1955, Jul. 2008.

[29] G. Todeschini and A. E. Emanuel, "Transient response of a wind energy conversion system used as active filter," IEEE Trans. Energy Convers., vol. 26, no. 2, pp. 522-531, Jul. 2011.

[30] M. Bollen and S. Ronnberg, "Primary and secondary harmonics emission; harmonic interaction-a set of definitions," in Proc. Int. Conf. Harmon. Qual. Power, Belo Horizonte, Brazil, 2016, pp. 703-708.

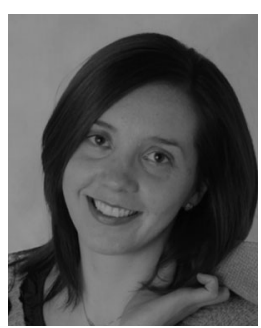

Grazia Todeschini (S'07-M'10-SM'14) received the B.Sc. and M.Sc. degrees in electrical engineering from the Politecnico di Milano, Milano, Italy, in 2003 and 2005, respectively, and the Ph.D. degree in electrical and computer engineering from Worcester Polytechnic Institute, Worcester, MA, USA, in 2010. She was a Senior Consultant with the EnerNex, Knoxville, TN, USA, and a Senior Power Studies Engineer with the General Electric, Philadelphia, PA, USA. She became a Senior Lecturer with Swansea University, Swansea, U.K., in 2016. Her research interests include power quality, power converter modeling, renewable energy integration, and power system analysis.

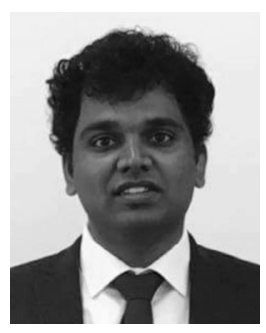

Senthooran Balasubramaniam (S'12-M'18) received the B.Eng. (Hons.) degree in electronic engineering from the University of Surrey, Guildford, U.K., in 2012, and the Ph.D. degree in electrical and electronic engineering from Cardiff University, Cardiff, U.K., in 2017. He is currently a Postdoctoral Researcher with the Energy Safety Research Institute, Swansea University, Swansea, U.K. His research interests include HVdc technologies, power electronics, grid integration of renewable energy, and power system control and stability.

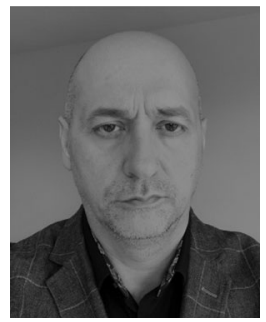

Petar Igic (SM'15) received the Dipl.-Eng. and Mag.Sc. degrees from the University of Nis, Serbia, in 1993 and 1997, respectively, and the Ph.D. degree from Swansea University, Swansea, U.K., in 2000. $\mathrm{He}$ is currently a Professor in power semiconductor applications with Coventry University, Coventry, UK. His research interests include power semiconductor applications and characterization of microelectronic power semiconductor devices. 